OPEN ACCESS

Edited by:

Tae-Hee Cho,

Hospices Civils de Lyon, France

Reviewed by:

Silke Walter,

Saarland University Hospital, Germany Nikoloz Tsiskaridze,

Pineo Medical Ecosystem, Georgia

*Correspondence:

Juhani Ritvonen

juhani.ritvonen@helsinki.fi

Specialty section: This article was submitted to

Stroke,

a section of the journal

Frontiers in Neurology

Received: 07 February 2021 Accepted: 30 March 2021

Published: 04 May 2021

Citation:

Ritvonen J, Sairanen T, Silvennoinen H, Virtanen P, Salonen O, Lindsberg PJ and Strbian D (2021)

Comatose With Basilar Artery Occlusion: Still Odds of Favorable Outcome With Recanalization Therapy. Front. Neurol. 12:665317. doi: 10.3389/fneur.2021.665317

\section{Comatose With Basilar Artery Occlusion: Still Odds of Favorable Outcome With Recanalization Therapy}

\author{
Juhani Ritvonen ${ }^{1 *}$, Tiina Sairanen ${ }^{1,2}$, Heli Silvennoinen ${ }^{3}$, Pekka Virtanen $^{3}$, Oili Salonen ${ }^{3}$, \\ Perttu J. Lindsberg ${ }^{1,2}$ and Daniel Strbian ${ }^{1,2}$ \\ ${ }^{1}$ Clinical Neurosciences, University of Helsinki, Helsinki, Finland, ${ }^{2}$ Neurological Research Unit, Department of Neurology, \\ Neurocenter, Helsinki University Hospital, Helsinki, Finland, ${ }^{3}$ Helsinki Medical Imaging Center, Helsinki University Hospital, \\ University of Helsinki, Helsinki, Finland
}

Background: Around 30-60\% of patients with basilar artery occlusion (BAO) present with coma, which is often considered as a hallmark of poor prognosis.

Aim: To examine factors that will help predict outcomes in patients with BAO comatose on admission.

Methods: A total of 312 patients with angiography-proven BAO were analyzed. Comas were assessed as Glasgow Coma Scale (GCS) of $\leq 8$ or impaired level of consciousness ascertained in the medical records. Outcomes were evaluated with the modified Rankin Scale (mRS) over a phone call at 3 months. In our study, 53 patients were excluded due to inadequate data on the level of consciousness.

Results: In total, 103/259 (39.8\%) of BAO patients were comatose on admission. Factors associated with acute coma were higher age, coronary artery disease, convulsions, extent of early ischemia by posterior circulation Acute Stroke Prognosis Early CT Score (pc-ASPECTS) < 8, absence of patent posterior collateral vasculature, and occlusion over multiple segments of BA. A total of 21/103 (20.4\%) of comatose patients had a favorable outcome (mRS 0-3), and 12/103 (11.7\%) had a good outcome (mRS 0-2). Factors associated with a favorable outcome in comatose BAO patients were younger age $(p=0.010)$, less extensive baseline ischemia $(p=0.027)$, recanalization $(p=0.013)$, and avoiding symptomatic intracranial hemorrhage $(\mathrm{SICH})(p=0.038)$. Factors associated with the poorest outcome or death (mRS 5-6) were older age $(p=0.001)$, diabetes $(p=0.022)$, atrial fibrillation $(p=0.016)$, lower median GCS [4 (IQR 3.6) vs. 6 (5-8); $p=0.006$ ], pc-ASPECTS $<8$ ( $p=0.003$ ), unsuccessful recanalization $(p=0.006)$, and $\mathrm{sICH}(p=0.010)$. Futile recanalization (mRS $4-6)$ was significantly more common in comatose patients (49.4 vs. $18.5 \%, p<0.001$ ).

Conclusions: One in five BAO patients with acute coma had a favorable outcome. Older patients with cardiovascular comorbidities and already existing ischemic lesions before reperfusion therapies tended to have a poor prognosis, especially if no recanalization is achieved and $\mathrm{sICH}$ occurred.

Keywords: basilar artery, outcome, coma, stroke, recanalization therapy 


\section{INTRODUCTION}

Basilar artery occlusion (BAO) accounts for $1 \%$ of all ischemic strokes but is still often considered the most appalling form of stroke $(1,2)$. The clinical picture of BAO varies greatly, and roughly $30-60 \%$ of patients present with the most shocking state, being comatose on admission (3-10). This initial coma or imminent locked-in state results typically from dense pontine ischemia, which anatomically encompasses essential areas forming the reticular activating system responsible for sustaining consciousness and motor pyramidal tracts $(1,11)$.

Indeed, in the face of the poor outcome reported to be associated with progressing pontine ischemia and depressed consciousness, acute coma in BAO, a discrete clinical entity, is one of the most challenging emergencies to be coped with by the emergency department (ED) attending neurologists and other acutologists $(6,12)$. However, it has also been reported that, with successful recanalization therapy, up to $15-26 \%$ of these patients presenting comatose on admission will eventually have a favorable outcome [modified Rankin Scale (mRS) 0-3] $(6,13,14)$.

Which clinical signs, imaging characteristics and presumptive biomarkers could be used to assist in early risk stratification supporting initial management decisions, i.e., aggressive or deescalated supportive therapy, and guide pressing interactions with the close ones? Mortality among intubated and mechanically ventilated stroke patients is high, yet the treatment decisions are not simple. It is one of the greatest challenges in the acute setting to estimate the prognostic potential to life "worth living" and the extent of treatments worth giving (15). Coma on presentation has been one negative prognostic marker to influence such decisions, especially regarding invasive therapies (4). Neurologists managing these patients often lack urgent directions in making these fundamental care decisions, for which this analysis was performed.

BAO carries high mortality of up to $95 \%$ if recanalization does not occur $(1,2,7)$. Pallesen et al. (6) reported brainstem ischemia to be an important factor in predicting mortality in comatose BAO patients, which underlines the need for the early reversal of BAO with intravenous thrombolysis (IVT) or endovascular treatment (EVT). However, according to our knowledge, the baseline characteristics potentially predicting the outcome after $\mathrm{BAO}$ patients presenting with coma have never been systemically examined. This led us to carry out the present investigation in our sizable consecutive cohort of BAO patients treated with IVT and/or EVT.

\section{AIM OF THE STUDY}

We set out to examine factors that would help in predicting the outcome in BAO patients presenting with acute coma on admission.

\section{METHODS}

\section{Patients}

A total of 312 consecutive patients with angiography-proven BAO, treated between June 1995 and September 2019 in the
Department of Neurology, Helsinki University Hospital, were analyzed. Coma was assessed as Glasgow Coma Scale (GCS) of $\leq 8$ either in emergency medical service (EMS) records or at $\mathrm{ED}$ (whichever is lowest) before initiation of recanalization therapy. In subjects where GCS was not routinely reported, the level of consciousness was ascertained from the medical records describing the detailed responsiveness of the patient. In essence, the patient was defined as "comatose" if the level of consciousness was documented to be clearly impaired or if there was a need for intubation due to a low level of consciousness. We do performed endovascular intervention in the majority of the cases under conscious sedation, and if a patient was intubated only to perform neurointerventional therapy or neuroimaging, and not because of the low level of consciousness, the patient was not defined as "comatose." As the level of consciousness on presentation could not be ascertained reliably due to insufficient EMS records and/or patient medical records, 53 patients were excluded.

Patient data, including also the 3-month follow-up assessment, were collected as a part of routine hospital care, and approval from an ethics committee or informed consent were thus not required to collect and reconcile retrospective clinical data in our institution. National Institutes of Health Stroke Scale (NIHSS) score is obtained for all acute recanalization patients on admission but since its determination may be interfered with by a low level of consciousness and possible anesthetic sedation, we excluded it from the analyses. The BAO "phenotype" was defined as a sudden or progressive onset of symptoms, as previously described (1).

\section{Imaging}

Imaging was performed on admission for all patients with either computed tomography (CT) or magnetic resonance imaging (MRI) accompanied by CT angiography (CTA), MR angiography (MRA), or digital subtraction angiography (DSA). Control imaging was obtained $\sim 24 \mathrm{~h}$ after treatment and whenever clinical deterioration occurred. The extent of baseline ischemia was evaluated with the posterior circulation Acute Stroke Prognosis Early CT Score (pc-ASPECTS) (16) (Figure 1).

Occlusion length was dichotomized to either "long," consisting of two or more basilar artery segments (proximal, middle, and distal), or "short," consisting of one segment. Whether occlusion extended to the vertebral artery (VA) was also analyzed. Recanalization was assessed from controlled angiography images and dichotomized as partial to complete [thrombolysis in myocardial infarction (TIMI) score 2-3] or nil to minimal (TIMI 0-1) (17). Futile recanalization was defined as successful recanalization with no clinical benefit demonstrated as an mRS score $4-6$ at 3 months (18).

Collateral vasculature was assessed from baseline angiography images by identifying the presence and number of posterior communicating arteries (PCom) and the presence of at least one superior cerebellar artery (SCA) and posterior cerebral artery (PCA). Symptomatic intracranial hemorrhage (sICH) was defined according to European Cooperative Acute Stroke Study II (ECASS II) criteria (19). Radiological data were analyzed by an experienced neuroradiologist (HS/PV/OS). 

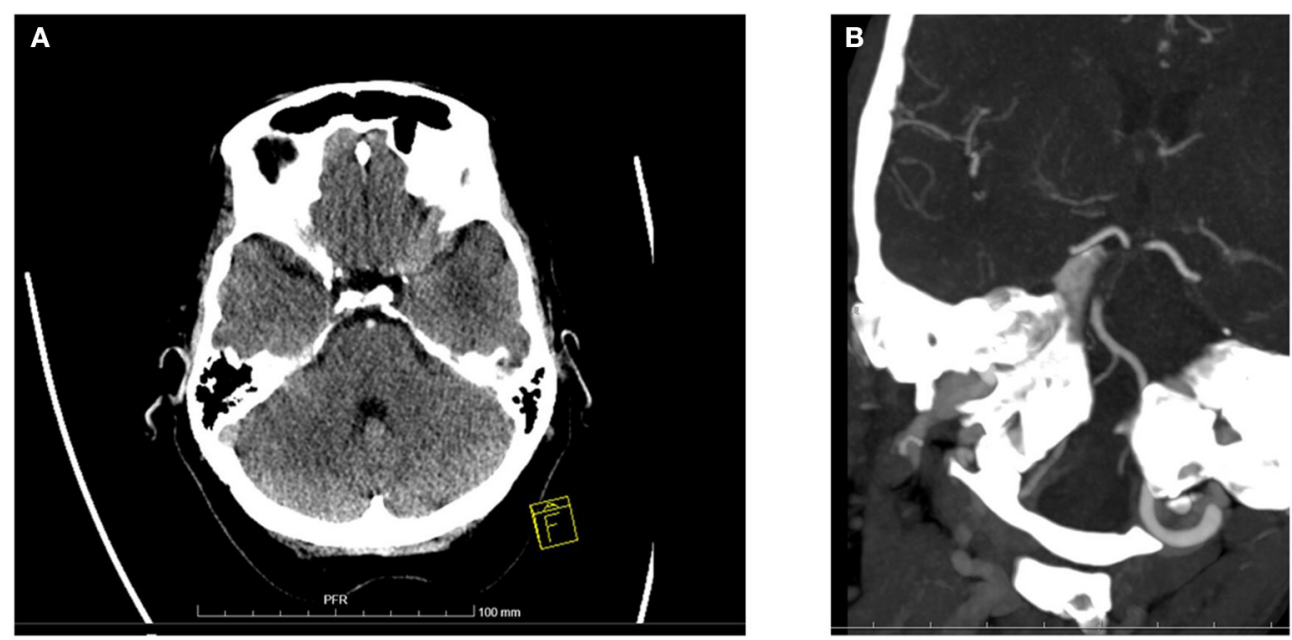

FIGURE 1 | Baseline ischemic changes and basilar artery occlusion in computer tomography. (A) A 69-year-old woman was comatose on admission (GCS 4) and diagnosed with basilar artery occlusion. This baseline CT scan shows extensive ischemic changes in both cerebellar hemispheres and bilaterally in pons. Overall pc-ASPECTS was 5. A dense basilar artery is also seen. Patient received IVT but died on day 3 of hospitalization. (B) A 65-year-old woman was comatose on admission (GCS 6). Baseline CTA shows long BAO over mid- and caudal BA. IVT was administered and subsequent mechanical thrombectomy was performed with successful recanalization. Patient recovered with a 3-month mRS of 1.

\section{Outcome}

The outcome was measured with an mRS obtained at 3 months by a certified neurologist. It was then dichotomized as favorable ( $\mathrm{mRS}$ 0-3) vs. poor (mRS 4-6). An mRS score of 3 was considered still favorable due to the abysmal natural course of BAO, and this is unlike anterior circulation, where an mRS $0-2$ is most often regarded as a favorable outcome. Secondary outcome measures were a good outcome (mRS 0-2) and being bedridden or dead (mRS 5-6) at 3 months. Mortality was also analyzed.

\section{Statistical Analyses}

Dichotomous variables were analyzed with Pearson's chi-square test or Fisher's exact test when appropriate. The distribution of continuous variables was tested for normality and analyses were performed with $t$-test for normally distributed or with Mann-Whitney U-test for non-normally distributed variables. Multivariable analysis of factors associated with coma was made with a stepwise backward logistic regression model, and variables with probability $(p)$ value $<0.1$ in univariate model were included in order to avoid selection bias and overfitting. For factors associated with favorable outcomes in comatose patients, no multivariable model was possible to construct due to the small number of subjects. Therefore, only the univariate model was built. SPSS 25 (IBM, Armonk, NY, USA) was used in all statistical analyses.

\section{RESULTS}

Among the cohort of 259 BAO patients, 103 (39.8\%) presented comatose (GCS $\leq 8$ ) on admission. 85/103 (82.5\%) of comatose patients were intubated. For all of them, the reason for intubation was a poor clinical condition or securing the airway because of impaired consciousness. In total, 35 patients (13.5\%) had baseline imaging with MRI, and the remaining majority underwent CT scans. Patient characteristics are shown in Table $\mathbf{1 .}$ Comatose patients tended to be older, had more frequent histories of coronary artery disease and myocardial infarctions, and were more likely to present with convulsions. Regarding brain imaging findings of the posterior circulation area, patients with initial coma had more often extensive baseline ischemic lesions as evaluated with pc-ASPECTS $<8$ (29.1 vs. $11.6 \%$, $p<0.001)$ and more often radiological signs of bilateral ischemia (35.3 vs. $14.5 \%, p<0.001$ ).

In vascular imaging of the posterior circulation, collateral circulation was less complete in comatose patients as compared to non-comatose patients with absence of patent PComs of the circle of Willis in 29.0 vs. $13.6 \%(p=0.003)$ and absence of SCAs in 42.0 vs. $22.4 \%$ ( $p=0.001)$, respectively. Comatose patients also had multiple segments $(\geq 2)$ of BA occluded and a higher incidence of occlusions extending to the vertebral artery (VA) (Table 1).

There was no difference in the rate of EVT or IVT between comatose and non-comatose patients, and the vast majority underwent IVT (90.3\%). There were only three patients not treated with recanalization therapy. In detail, there was one patient with spontaneous recanalization in DSA and 3-month mRS 3, one patient with EVT attempt but no successful thrombectomy due to difficult stenosis and no access with thrombectomy device (mRS 6), and another with only IVT bolus administered but discontinued infusion due to a history of malignancy (mRS 4). Treatment delays did not differ between comatose and non-comatose BAO patients (Table 1).

In multivariable analysis (Table 2) factors independently associated with acute coma in BAO, patients were of older age, had a history of coronary artery disease, convulsions, presence 
TABLE 1 | Characteristics of comatose vs. non-comatose patients at presentation.

\begin{tabular}{|c|c|c|c|c|}
\hline$n(\%) / m e d i a n(I Q R)$ & All $(n=259)$ & Non-comatose $(n=156,60.2 \%)$ & Comatose $(n=103,39.8 \%)$ & $p$ \\
\hline \multicolumn{5}{|c|}{ Demographic and clinical characteristics } \\
\hline Male sex & $169(65.3 \%)$ & $106(67.9 \%)$ & $63(61.2 \%)$ & 0.262 \\
\hline Age (years) & $68(59-77)$ & $64(58-75)$ & $73(63-79)$ & $<0.001$ \\
\hline Diabetes & $42(16.2 \%)$ & $24(15.4 \%)$ & $18(17.5 \%)$ & 0.655 \\
\hline Atrial fibrillation & $60(23.2 \%)$ & $31(19.9 \%)$ & $29(28.2 \%)$ & 0.122 \\
\hline Hypertension & 149 (57.5\%) & $85(54.5 \%)$ & $64(62.1 \%)$ & 0.223 \\
\hline Dyslipidemia & $106(40.9 \%)$ & $64(41.0 \%)$ & $42(40.8 \%)$ & 0.968 \\
\hline Chronic heart failure & $22(8.5 \%)$ & $10(6.4 \%)$ & $12(11.7 \%)$ & 0.139 \\
\hline Coronary artery disease & $46(17.8 \%)$ & $21(13.5 \%)$ & $25(24.3 \%)$ & 0.026 \\
\hline History of myocardial infarction & $26(10.0 \%)$ & $11(7.1 \%)$ & $15(14.6 \%)$ & 0.049 \\
\hline Previous stroke & $63(24.3 \%)$ & $38(24.4 \%)$ & $25(24.3 \%)$ & 0.987 \\
\hline Sudden onset phenotype & $214(82.6 \%)$ & 125 (80.1\%) & 89 (86.4\%) & 0.192 \\
\hline Prodromal symptoms & $56(21.6 \%)$ & 37 (23.7\%) & 19 (18.4\%) & 0.313 \\
\hline Convulsions & $37(14.3 \%)$ & $12(7.7 \%)$ & $25(24.3 \%)$ & $<0.001$ \\
\hline \multicolumn{5}{|l|}{ Radiological parameters } \\
\hline pc-ASPECTS $<8$ & $48(18.6 \%)$ & $18(11.6 \%)$ & $30(29.1 \%)$ & $<0.001$ \\
\hline Bilateral ischemia & $58(22.8 \%)$ & $22(14.5 \%)$ & $36(35.3 \%)$ & $<0.001$ \\
\hline Brainstem ischemia & 48 (18.9\%) & 24 (15.8\%) & $24(23.5 \%)$ & 0.122 \\
\hline No patent PComs & 49 (19.8\%) & 20 (13.6\%) & $29(29.0 \%)$ & 0.003 \\
\hline 2 PComs & $132(53.4 \%)$ & 89 (60.5\%) & $43(43.0 \%)$ & 0.007 \\
\hline At least one patent PCA & 233 (94.3\%) & 138 (93.9\%) & 95 (95.0\%) & 0.708 \\
\hline At least one patent SCA & $172(69.6 \%)$ & $114(77.6 \%)$ & $58(58.0 \%)$ & 0.001 \\
\hline Long $\mathrm{BAO}$ ( $\geq 2$ segments) & $100(40.0 \%)$ & $47(31.5 \%)$ & $53(52.5 \%)$ & 0.001 \\
\hline BAO exceeding to VA & $59(23.7 \%)$ & 27 (18.1\%) & 32 (32.0\%) & 0.012 \\
\hline \multicolumn{5}{|l|}{ Treatments } \\
\hline IVT & 234 (90.3\%) & 141 (90.4\%) & 93 (90.3\%) & 0.980 \\
\hline EVT & 75 (29.0\%) & 45 (28.8\%) & 30 (29.1\%) & 0.961 \\
\hline $\mathrm{IVT}+\mathrm{EVT}$ & 53 (20.5\%) & 32 (20.5\%) & 21 (20.4\%) & 0.981 \\
\hline \multicolumn{5}{|l|}{ Time delays (min) } \\
\hline OTा & 252 (138-720) & $238(125-780)$ & $282(158-640)$ & 0.334 \\
\hline SDT & $155(78-451)$ & $152(75-474)$ & $158(85-371)$ & 0.659 \\
\hline DTN & $51(25-195)$ & $85(38-180)$ & $65(30-185)$ & 0.060 \\
\hline
\end{tabular}

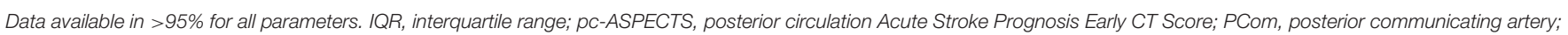

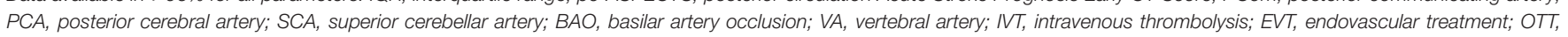
Onset-to-treatment time, SDT, symptom-to-door time; DTN, Door-to-needle time.

Statistically significant $p$-values $(<0.05)$ are bolded.

of extensive baseline ischemia, absence of PComs and SCAs, and long BA occlusion.

Initial coma was associated with poor outcome, and $79.6 \%$ of comatose patients had an mRS $4-6$ at 3 months compared to $38.4 \%$ among non-comatose patients $(p<0.001)$. Overall, $59.2 \%$ of comatose patients died within 3 months (Table 3 ), and $72.8 \%$ of patients with initial coma were either bedridden or died (mRS 5-6) at 3 months, which compared to the $28.5 \%$ of non-comatose patients $(p<0.001)$. Of the comatose patients, $13.6 \%$ had an mRS of 5 at 3 months. However, up to $20.4 \%$ of comatose patients still had a favorable outcome (mRS 0-3), and $11.7 \%$ had a good outcome of mRS $0-2$ at 3 months. There was no significant difference in rates of recanalization between comatose and non-comatose patients, but futile recanalization (mRS 4-6) was significantly more common in comatose patients ( 49.4 vs.
$18.5 \%, p<0.001)$. There was a trend toward a higher rate of posttreatment sICH in comatose patients (13.9 vs. $7.8 \%$ ), but this was not statistically significant $(p=0.117)$.

An exact GCS score was available for 83/103 of comatose patients. A total of 19 patients had GCSs scores of three. The GCS was four in 18 patients, five in 15 patients, six in 11 patients, and seven in 14 patients. Only six patients had GCS score of eight. In total, 3/19 (15.8\%) of patients with a GCS score of three had an mRS $0-3$ at 3 months. The rest of these patients $(16 / 19,84.2 \%)$ had the poorest outcome of an mRS of 5-6, and 13 of them (68.4\%) were dead at 3 months. Half of the patients with GCS score of eight had an mRS $0-3$ at 3 months, one died, and two had the poorest outcome (mRS 5-6).

Characteristics associated with favorable outcomes in patients with initial coma are shown in Table 4. Patients with favorable 
3-month outcome were younger [median age 65 (IQR 57-74) vs. $74(66-80)$ years, $p=0.010]$ and displayed less frequent extensive baseline ischemic lesions (pc-ASPECTS $<8$ in 9.5 vs. $34.1 \%, p=0.027$ ) compared with patients with an outcome mRS of 4-6. Other demographic or clinical characteristics did not differ between the outcomes of comatose patients, nor did the radiological surrogates of vascular collateralization. Recanalization was a necessary requirement for a favorable outcome (i.e., mRS 0-3) in all but two patients (in 19/21). None of the patients that achieved a favorable outcome had sICH (Table 4).

Factors predicting the worst possible outcome, projected being bedridden or dead, in a univariate model are shown in Table 5. Patients with a 3-month mRS 5-6 were older, presented with diabetes and atrial fibrillation more frequently, presented with lower median GCS score [median 4 (IQR 3-6) vs. 6 (5-7), $p=0.026]$, and had more often extensive baseline ischemic changes and bilateral ischemia in baseline imaging. All patients with acute coma and post-treatment sICH had an mRS 5-6 at 3 months (11 dead and 3 with an mRS of 5). The rate of

TABLE 2 | Factors associated with acute coma in BAO patients.

\begin{tabular}{lccc}
\hline & OR & 95\% C.I. & $\boldsymbol{p}$ \\
\hline Age (per year) & 1.04 & $1.01-1.06$ & 0.004 \\
CAD & 2.18 & $1.01-4.74$ & 0.049 \\
Convulsions & 4.96 & $2.12-11.61$ & $<0.001$ \\
pc-ASPECTS $<8$ & 3.64 & $1.74-7.62$ & 0.001 \\
Absence of patent PComs & 2.44 & $1.17-5.09$ & 0.018 \\
At least one patent SCA & 0.40 & $0.21-0.75$ & 0.004 \\
Long BAO ( $\geq 2$ segments) & 2.55 & $1.39-4.67$ & 0.002 \\
\hline
\end{tabular}

CAD, coronary artery disease; pc-ASPECTS, posterior circulation Acute Stroke Prognosis Early CT Score; PCom, posterior communicating artery; SCA, superior cerebellar artery; $B A O$, basilar artery occlusion.

Factors with probability value $(p)<0.1$ in a univariate model (Table 1) were included in a backward stepwise logistic regression model. recanalization was lower in patients with the worst outcome (64.0 vs. $88.9 \%, p=0.006$ ). Extensive baseline ischemic changes were a major factor predicting the poorest outcome (mRS 5-6). In total, 30/103 $(29.1 \%)$ of the acutely comatose patients had pc-ASPECTS $<8$, and only two of them achieved favorable outcomes (mRS $0-3$ ). In terms of patients with acute coma and pc-ASPECTS $<8,93.3 \%(28 / 30)$ were bedridden or dead (mRS 5-6) after 3 months (Table 5). When patients with pc-APECTS $<8$ were excluded from analysis, $26.0 \%(19 / 73)$ of patients with initial coma achieved favorable outcome, and 64.4\% (47/73) had an mRS 5-6.

\section{DISCUSSION}

Approximately $40 \%$ of BAO patients were initially comatose, which is in line with rates of $30-60 \%$ reported in previous studies (3-10). The main observation of this study was that, despite the dismal presentation on admission, still 2/10 comatose BAO patients treated with recanalization therapy reached functionally meaningful survival. Coma vs. no coma on presentation was not associated with differences in receiving either IVT or EVT treatment, nor with the differences in onset to treatment or symptom to door times. Our data consists of a large cohort of BAO patients treated in our institution between 1995 and 2019. In view of data from RCTs published in 2015 (20) demonstrating the efficacy of EVT in large vessel occlusions in anterior circulation, EVT was adopted in more routine use only after this in 2015. As previously reported by us (21), our IVTtreated cohort had comparable outcome rates with many EVT cohorts, and therefore no evidence-based amendment toward EVT-based treatment strategy has been made in our written inhouse stroke management guidelines (Figure 2), although the rates of EVT have increased in recent years.

In our present cohort, older age, a history of coronary artery disease as a manifestation of atherosclerosis, convulsions at onset, extensive early ischemia in the vertebrobasilar region, and a lack of patent collateral vasculature of either circle of

TABLE 3 | Outcome measures between comatose vs. non-comatose patients.

\begin{tabular}{|c|c|c|c|c|}
\hline Outcome, $n(\%)$ & All $(n=259)$ & Non-comatose $(n=156,60.2 \%)$ & Comatose $(n=103,39.8 \%)$ & $p$ \\
\hline \multicolumn{5}{|l|}{ 90-day mRS } \\
\hline mRS 0-2 & $88(34.6 \%)$ & $76(50.3 \%)$ & $12(11.7 \%)$ & $<0.001$ \\
\hline mRS 0-3 & $114(44.9 \%)$ & $93(61.6 \%)$ & $21(20.4 \%)$ & $<0.001$ \\
\hline mRS 5-6 & $118(46.5 \%)$ & $43(28.5 \%)$ & 75 (72.8\%) & $<0.001$ \\
\hline Dead at 3 months & $98(38.3 \%)$ & $37(24.2 \%)$ & $61(59.2 \%)$ & $<0.001$ \\
\hline $\mathrm{slCH}$ & 26 (10.2\%) & $12(7.8 \%)$ & 14 (13.9\%) & 0.117 \\
\hline
\end{tabular}

${ }^{\dagger}$ Data available in $79 \%$.

${ }^{\ddagger}$ Data available in $78 \%$.

mRS, modified Rankin Scale; TIMI, Thrombolysis in Myocardial Infarction; s/CH, symptomatic intracerebral hemorrhage.


intracranial hemorrhage ( $\mathrm{S} / \mathrm{CH}$ ) was defined according to European Cooperative Acute Stroke Study II (ECASS II) criteria.

Statistically significant $p$-values $(<0.05)$ are bolded. 
TABLE 4 | Characteristics associated with favorable outcome in comatose BAO patients.

\begin{tabular}{|c|c|c|c|c|}
\hline$n(\%) /$ median (IQR) & All $(n=103)$ & mRS 0-3 $(n=21,20.4 \%)$ & mRS 4-6 $(n=82,79.6 \%)$ & $p$ \\
\hline \multicolumn{5}{|l|}{ Characteristics } \\
\hline Male sex & $63(61.2 \%)$ & $14(66.7 \%)$ & 49 (59.8\%) & 0.562 \\
\hline Age & $73(63-79)$ & $65(57-74)$ & $74(66-80)$ & 0.010 \\
\hline Diabetes & $18(17.5 \%)$ & $1(4.8 \%)$ & $17(20.7 \%)$ & 0.112 \\
\hline Atrial fibrillation & 29 (28.2\%) & $3(14.3 \%)$ & $26(31.7 \%)$ & 0.113 \\
\hline Chronic heart failure & $12(11.7 \%)$ & $4(19.0 \%)$ & $8(9.8 \%)$ & 0.259 \\
\hline Coronary artery disease & 25 (24.3\%) & $5(23.8 \%)$ & 20 (24.4\%) & 0.956 \\
\hline Previous myocardial infarction & $15(14.6 \%)$ & $3(14.3 \%)$ & $12(14.6 \%)$ & 1.000 \\
\hline Previous stroke & 25 (24.3\%) & $4(19.0 \%)$ & $21(25.6 \%)$ & 0.531 \\
\hline Sudden onset phenotype & 89 (86.4\%) & $18(85.7 \%)$ & $71(86.6 \%)$ & 1.000 \\
\hline \multicolumn{5}{|l|}{ Radiological parameters } \\
\hline pc-ASPECTS $<8$ & $30(29.1 \%)$ & $2(9.5 \%)$ & $28(34.1 \%)$ & 0.027 \\
\hline Bilateral ischemia & $36(35.3 \%)$ & $5(23.8 \%)$ & $31(38.3 \%)$ & 0.217 \\
\hline Brainstem ischemia & $24(23.5 \%)$ & $3(14.3 \%)$ & $21(25.9 \%)$ & 0.388 \\
\hline No patent PComs & $29(29.0 \%)$ & $6(30.0 \%)$ & $23(28.8 \%)$ & 0.912 \\
\hline 2 PComs & $43(43.0 \%)$ & $10(50.0 \%)$ & $33(41.3 \%)$ & 0.480 \\
\hline At least one patent PCA & 95 (95.0\%) & 19 (95.0\%) & 76 (95.0\%) & 1.000 \\
\hline At least one patent SCA & $58(58.0 \%)$ & $11(55.0 \%)$ & $47(58.8 \%)$ & 0.761 \\
\hline Long BAO ( $\geq 2$ segments) & 53 (52.5\%) & $11(55.0 \%)$ & 42 (51.9\%) & 0.801 \\
\hline BAO exceeding to VA & 32 (32.0\%) & $4(20.0 \%)$ & $28(35.0 \%)$ & 0.198 \\
\hline Recanalization (TIMI 2-3) & 57 (74.0\%) & 19(95.0\%) & $38(66.7 \%)$ & 0.013 \\
\hline SDT & $158(85-371)$ & $170(106-371)$ & $156(82-371)$ & 0.964 \\
\hline DTN & $85(38-180)$ & $89(51-165)$ & $85(38-189)$ & 0.902 \\
\hline
\end{tabular}

${ }^{\dagger}$ Data available in $81 \%$.

${ }^{\ddagger}$ Data available in $75 \%$.

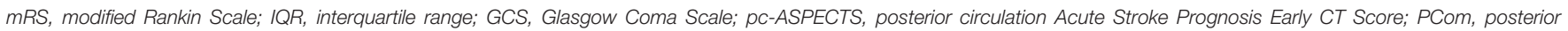

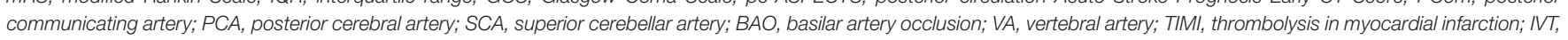
intravenous thrombolysis; EVT, endovascular treatment; OTT, Onset-to-treatment time; SDT, symptom-to-door time; DTN, Door-to-needle time.

Univariate model; a multivariable regression model was not constructed due to the small sample size.

Statistically significant $p$-values $(<0.05)$ are bolded.

Willis or cerebellum, besides long occlusions, were independently associated with acute coma in BAO patients. All of these have a logical neuroanatomical and neurophysiological explanation. Anatomically, the area vital to upholding consciousness is the pontine tegmentum, containing the ascending reticular activating system with a complex network of neurons $(1,11$, $22,23)$. Cerebrovascular atherosclerosis often leads to occlusions of the proximal and middle segments of the BA resulting in pontine ischemia and affecting areas upholding consciousness
(1). Convulsive-like movements and extension rigidity arise from pontine pyramidal tracts and are therefore suggestive of brainstem ischemia often involving also areas of reticular activating circuitry $(1,2)$. Posterior circulation (Pc) ASPECTS below 8 correlates well with the brainstem ischemia since $64.6 \%$ of patients with pc-ASPECTS $<8$ had brainstem ischemia either alone or combined with other VB-areas.

The observation that multisegmental occlusions of BA associated with initial coma are consistent with the probability 
TABLE 5 | Factors associated with poorest outcome (mRS 5-6) in comatose BAO patients.

\begin{tabular}{|c|c|c|c|c|}
\hline$n(\%) /$ mean (SD)/median (IQR) & All $(n=103)$ & mRS 0-4 $(n=28,27.2 \%)$ & mRS 5-6 ( $n=75,72.8 \%)$ & $p$ \\
\hline \multicolumn{5}{|l|}{ Characteristics } \\
\hline Male sex & $63(61.2 \%)$ & 19 (67.9\%) & $44(58.7 \%)$ & 0.395 \\
\hline Age & $71(12)$ & $65(14)$ & $73(10)$ & 0.001 \\
\hline Diabetes $n$ & $18(17.5 \%)$ & $1(3.6 \%)$ & $17(22.7 \%)$ & 0.022 \\
\hline Atrial fibrillation & $29(28.2 \%)$ & $3(10.7 \%)$ & 26 (34.7\%) & 0.016 \\
\hline Chronic heart failure & $12(11.7 \%)$ & $4(14.3 \%)$ & $8(10.7 \%)$ & 0.731 \\
\hline Coronary artery disease & 25 (24.3\%) & $7(25.0 \%)$ & $18(24.0 \%)$ & 0.916 \\
\hline Previous myocardial infarction & $15(14.6 \%)$ & $3(10.7 \%)$ & $12(16.0 \%)$ & 0.754 \\
\hline Previous stroke & 25 (24.3\%) & $6(21.4 \%)$ & $19(25.3 \%)$ & 0.681 \\
\hline Sudden onset phenotype & $89(86.4 \%)$ & $25(89.3 \%)$ & 64 (85.3\%) & 0.753 \\
\hline \multicolumn{5}{|l|}{ Radiological parameters } \\
\hline pc-ASPECTS $<8$ & $30(29.1 \%)$ & $2(7.1 \%)$ & $28(37.3 \%)$ & 0.003 \\
\hline Bilateral ischemia & $36(35.3 \%)$ & $5(17.9 \%)$ & 31 (41.9\%) & 0.023 \\
\hline Brainstem ischemia & $24(23.5 \%)$ & $3(10.7 \%)$ & $21(28.4 \%)$ & 0.061 \\
\hline No patent PComs & $29(29.0 \%)$ & $9(33.3 \%)$ & $20(27.4 \%)$ & 0.561 \\
\hline 2 PComs & $43(43.0 \%)$ & $13(48.1 \%)$ & $30(41.1 \%)$ & 0.527 \\
\hline At least one patent PCA & 95 (95.0\%) & 25 (92.6\%) & 70 (95.9\%) & 0.610 \\
\hline At least one patent SCA & $58(58.0 \%)$ & $14(51.9 \%)$ & $44(60.3 \%)$ & 0.449 \\
\hline Long BAO ( $\geq 2$ segments) & 53 (52.5\%) & $14(51.9 \%)$ & 39 (52.7\%) & 0.940 \\
\hline BAO exceeding to VA & 32 (32.0\%) & $6(22.2 \%)$ & 26 (35.6\%) & 0.202 \\
\hline Recanalization (TIMI 2-3) & $57(74.0 \%)$ & $25(92.6 \%)$ & $32(64.0 \%)$ & 0.006 \\
\hline SDT & $158(85-371)$ & 159 (98-368) & $158(82-451)$ & 0.961 \\
\hline DTN & $85(38-180)$ & $69(38-127)$ & $89(38-214)$ & 0.216 \\
\hline
\end{tabular}

${ }^{\dagger}$ Data available in $81 \%$.

${ }^{\ddagger}$ Data available in $75 \%$.

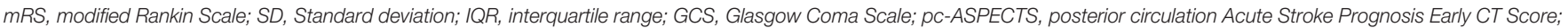

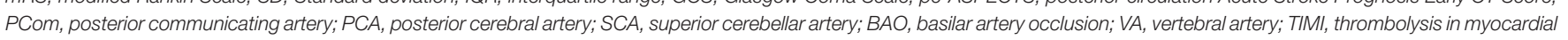
infarction; IVT, intravenous thrombolysis; EVT, endovascular treatment; OTT, Onset-to-treatment time; SDT, symptom-to-door time; DTN, Door-to-needle time.

Univariate model; a multivariable regression model was not possible to construct due to the small sample size.

Statistically significant p-values $(<0.05)$ are bolded.

of a greater number of perforating pontine arteries lacks patency on presentation. In line with these findings, both Ferbert et al. (3) and Cross et al. (24) reported a clot location most often in either proximal or middle segment of BA in comatose BAO patients. The finding that collateral status associated with coma on presentation is consistent with the concept of reverse "backflow" from the PComs and SCAs supplying circulation in the arterial branches distal to BAO maintaining brainstem vitality for longer periods of time (25). Greater collateral status has been reported to be associated with a better prognosis $(26,27)$.
The rates of 35 and $45 \%$ of good (mRS $0-2$ ) and favorable outcomes (mRS 0-3), respectively, among the whole BAO cohort (Table 3) are in line with or slightly better than in previous cohorts $(10,12,14,18,27-30)$. Poorer outcomes were significantly more frequent in comatose patients (Table 3 ). Multiple previous studies report unconsciousness as a predictor of poor outcome $(6,12,14,31-33)$, while others have failed to demonstrate such correlation reaching statistical significance (4, $5,9,10,24)$. This might be in part explained by the small number of study subjects and varying patient selection. When ischemic 


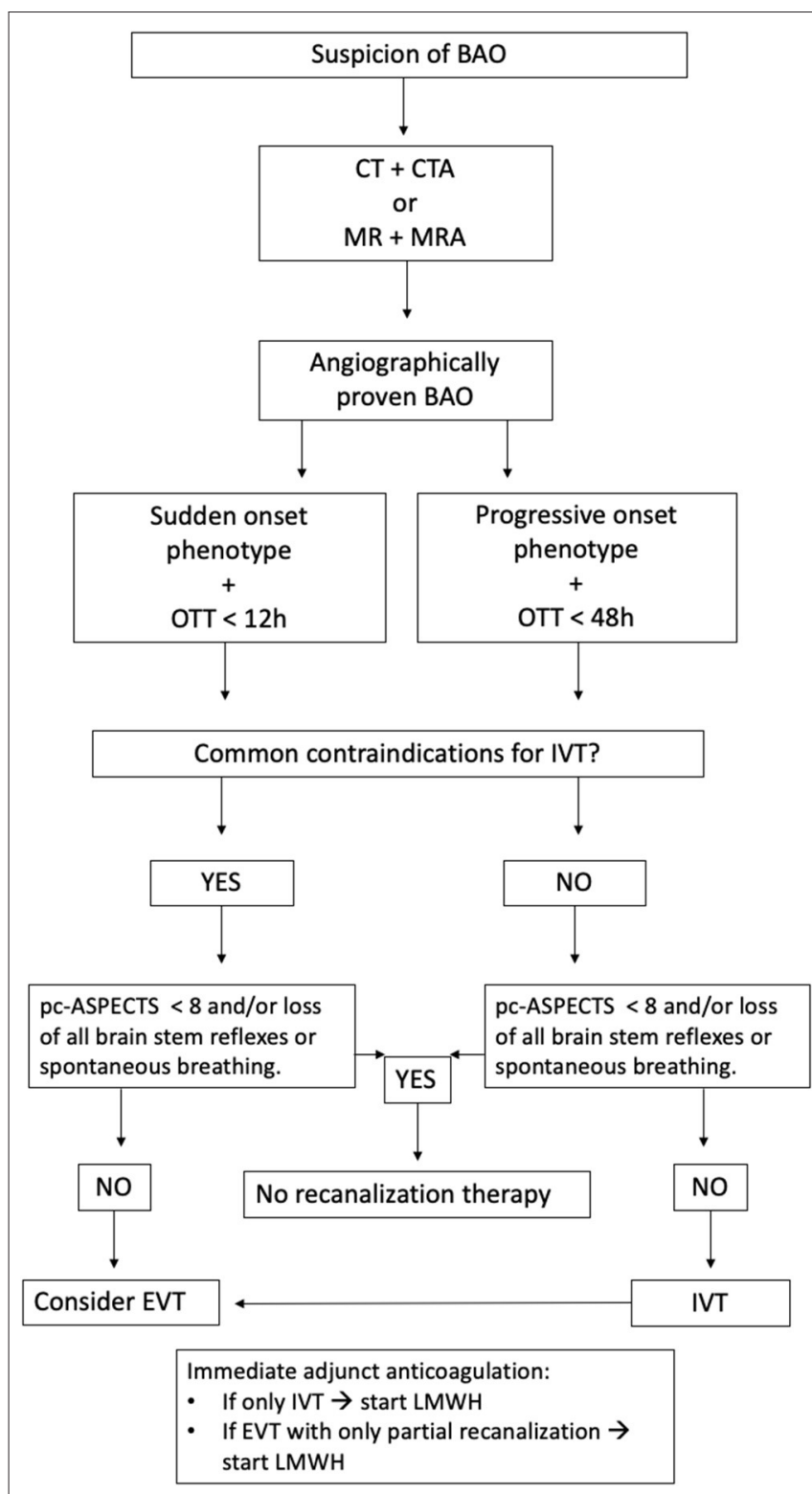

FIGURE 2 | Simplified flow chart on BAO treatment. BAO, Basilar artery occlusion; OTT, Onset-to-teatment time; IVT, Intravenous thrombolysis; pc-ASPECTS, posterior circulation Acute Stroke Prognosis Early CT Score; EVT, Endovascular treatment; LMWH, Low-molecular-weight heparin.

stroke patients requiring ventilation support were analyzed, GCS $<10$ on admission was an independent predictor of both early and late mortality (34). However, in the present study favorable outcome (3-month mRS 0-3) was achieved in one-fifth of BAO patients with initial coma. Similar results have been previously reported in the BASICS registry with a rate of good outcome of $17 \%$ in intraarterial thrombolysis (IAT) and $26 \%$ in IVT treated BAO patients with coma, tetraplegia, or locked-in state on presentation (13). In our previous smaller study, we found a favorable outcome (mRS $0-3$ ) in $22.5 \%$ of $\mathrm{BAO}$ patients needing intubation and mechanical ventilation (14). These rates are slightly higher than those reported by Pallesen et al. (6) with
$15.4 \%$ of comatose BAO patients achieving a favorable outcome (mRS 0-3). This might be partly explained with a relatively high number of patients with no recanalization therapy in their study since $33 \%$ of comatose patients did not receive any recanalization therapy, $38 \%$ underwent IAT, $4 \%$ were treated with IVT, and $24 \%$ were treated with IVT + IAT.

Since reduced consciousness in the case of BAO arises from brainstem ischemia that might be reversed with rapid recanalization therapy, it is conceivable that even a patient with acute coma can be recovered if permanent brainstem tissue damage remains limited. Indeed, Chandra and coworkers (4) reported that low GCS scores did not correlate with poor neurologic outcomes in patients with acute BAO managed with intraarterial therapy (4). Furthermore, Nagel et al. (9) reported that lower GCS was associated with less favorable outcome but when the multivariable model was construed, only pc-ASPECTS remained as an independent predictor of outcome (9). Similarly, $96 \%$ of patients with pc-ASPECTS $<8$ at baseline had poor outcomes (3-month mRS 3-6) in our previous study (35). In the present study, $93 \%$ of patients with acute coma and pc-ASPECTS $<8$ ended up dead or bedridden at 3 months. With further simulation of the current cohort, it was observed that, when BAO patients with low pc-ASPECTS were excluded from the analysis, the rate of favorable outcome raised to $26 \%$ within comatose patients, and the rate of the most devastating outcome of mRS 5-6 reduced to $64 \%$ (vs. $72.8 \%$ ). This supports our present inhouse treatment guideline in which extensive brainstem ischemia is a contraindication for $\mathrm{BAO}$ recanalization treatment but not coma as such (Figure 2). Since the cohort includes patients also from a period prior to the European label for IVT use in acute stroke, the experience and adherence to in-house protocol for BAO treatment has improved over the years (21).

Besides the absence of ischemic changes, factors associated with favorable outcomes in comatose BAO patients included younger age, successful recanalization, and avoiding posttreatment sICH, the latter two of which are treatment-related efficacy and safety goals worth further honing. On the other hand, additional factors associated with the poorest outcome (mRS 5-6) were older age, a burden of cardiovascular comorbidities (i.e., diabetes and atrial fibrillation), being deeply comatose, and imaging showing extensive ischemic changes. Although several of these factors have previously been shown to predict outcome after $\mathrm{BAO}(12,14,28-30,36,37)$, the relative predictive value of these factors projecting poor and favorable outcomes of initially comatose patients has not been described in detail due to relatively small study populations and cohort sizes of BAO. We remain with the clinical challenge of weighing each of these in decision making, taking all of them into consideration in each BAO patient.

An acutely comatose BAO patient is one of the most challenging emergencies in neurological ED evaluation. After the correct diagnosis has been reached, physicians need to conduct risk stratification to support decision making about the aggressiveness of the immediate therapy. The prognosis of $\mathrm{BAO}$ without recanalization is abysmal $(1,2)$, but the rate of futile recanalization is also high; roughly $50 \%$ of comatose patients in the present study did not avoid institutionalized living 
conditions or death despite success in recanalization. Mortality among mechanically ventilated stroke patients is known to be high, yet some of these patients do survive, occasionally even with no or only slight disability (15). It is known that physicians tend to be overly pessimistic in predicting survival and quality of life in patients with severe stroke or other critical illness and that many physicians' personal mindsets also affect their treatment decisions (15). This should be acknowledged in order to avoid a self-fulfilling prophecy. On the other hand, novel endovascular recanalization procedures and life-sustaining therapies in intensive care and stroke units are considerably expensive when given futilely to reach excellent vascular patency in patients projected toward a desperate prognosis. Avoiding sheer mortality should not be the steadfast main goal, and the probability of meaningful recovery with an acceptable quality of life should always be kept in mind when making treatment decisions. Therefore, every evidence-based assistance available is invaluable for physicians treating these infrequent BAO patients. This underlines the importance of our present evaluation of factors that might facilitate the challenging risk stratification and decision making in the acute setting.

Limitations of the present study are the relatively restricted number of subjects and the incomplete availability of radiological recanalization data for all patients, especially the ones with early clinical deterioration or death within $24 \mathrm{~h}$. Due to the small number of comatose patients, no multivariable regression models regarding their outcome were possible to construe. However, we report data from a previously wellestablished single-center cohort of an experienced stroke unit with a long-honed recanalization therapy protocol for BAO $(5,7,14,21,30,35,38)$.

In conclusion, one-fifth of $\mathrm{BAO}$ patients presenting with initial coma achieved a favorable outcome within 3 months. These patients were younger and lacking extensive baseline ischemic lesions of the vertebrobasilar region on baseline brain imaging. Therefore, unconsciousness per se and seemingly bleak clinical conditions upon presentation should not as such exclude $\mathrm{BAO}$ patients from receiving aggressive recanalization therapy.

\section{REFERENCES}

1. Arnold M, Mattle HP, Arnold M, Lindsberg J, Schonewille WJ, Schroth G. Basilar artery occlusion. Lancet Neurol. (2011) 10:1002-14. doi: 10.1016/S1474-4422(11)70229-0

2. Lindsberg PJ, Sairanen T, Strbian D, Kaste M. Current treatment of basilar artery occlusion. Ann NY Acad Sci. (2012) 1268:35-44. doi: 10.1111/j.1749-6632.2012.06687.x

3. Ferbert A, Brückmann H, Drummen R. Clinical features of proven basilar artery occlusion. Stroke. (1990) 21:1135-42. doi: 10.1161/01.STR.21.8.1135

4. Chandra RV, Law CP, Yan B, Dowling RJ, Mitchell PJ. Glasgow coma scale does not predict outcome post-intra-arterial treatment for basilar artery thrombosis. AJNR Am J Neuroradiol. (2011) 32:576-80. doi: 10.3174/ajnr.A2325

5. Lindsberg PJ, Soinne L, Tatlisumak T, Roine RO, Kallela M, Häppölä $\mathrm{O}$, et al. Long-term outcome after intravenous thrombolysis of basilar artery occlusion. JAMA. (2004) 292:1862-6. doi: 10.1001/jama.292. 15.1862
Rather, initial and post-treatment decisions during subsequent days should be made individually, considering prognostic key baseline characteristics, where the volume and location of infarction should be dominant in assisting maintenance or deescalation of intensive care $(9,39)$. These characteristics can be brought up in discussions with the closest ones and when making decisions regarding the prolongation of intensive care unit treatment.

\section{DATA AVAILABILITY STATEMENT}

The original contributions presented in the study are included in the article/supplementary material, further inquiries can be directed to the corresponding author/s.

\section{AUTHOR CONTRIBUTIONS}

JR, TS, PL, and DS contributed to the design of the study and the writing process of the manuscript. HS, OS, and PV collected the radiological data. JR performed statistical analyses. All authors contributed to manuscript revision, read, and approved the submitted version.

\section{FUNDING}

Research support funding has been received from the Sigrid Jusélius Foundation, Jane and Aatos Erkko Foundation, and Helsinki University Hospital Governmental Research Funding to our research group.

\section{ACKNOWLEDGMENTS}

The authors gratefully acknowledge the whole staff of the Department of Neurology and Imaging Center in Helsinki University Hospital and the personnel of the Clinical Neurology research unit for their work for this project.
6. Pallesen LP, Khomenko A, Dzialowski I, Barlinn J, Barlinn K, Zerna C, et al. CT-angiography source images indicate less fatal outcome despite coma of patients in the Basilar Artery International Cooperation Study. Int J Stroke. (2016) 12:145-51. doi: 10.1177/1747493016669886

7. Lindsberg PJ, Mattle HP. Therapy of basilar artery occlusion: a systematic analysis comparing intra-arterial and intravenous thrombolysis. Stroke. (2006) 37:922-8. doi: 10.1161/01.STR.0000202582.29510.6b

8. Hacke W, Zeumer H, Ferbert A, Briickmann H, del Zoppo GJ. Intra-arterial thrombolytic therapy improves outcome in patients with acute vertebrobasilar occlusive disease. Stroke. (1988) 19:1216-22. doi: 10.1161/01.STR.19.10.1216

9. Nagel S, Herweh C, Köhrmann M, Huttner HB, Poli S, Hartmann M, et al. MRI in patients with acute basilar artery occlusion - DWI lesion scoring is an independent predictor of outcome. Int J Stroke. (2011) 7:282-8. doi: 10.1111/j.1747-4949.2011.00705.x

10. Mourand I, Machi P, Nogué E, Arquizan C, Costalat V, Picot M-C, et al. Diffusion-weighted imaging score of the brainstem: a predictor of outcome in acute basilar artery occlusion treated with the Solitaire FR device. AJNR Am J Neuroradiol. (2014) 35:1117-23. doi: 10.3174/ajnr.A3870 
11. Nikic P, Jovanovic D, Paspalj D, Georgievski-Brkić B, Savić M. Clinical characteristics and outcome in the acute phase of ischemic locked-in syndrome: case series of twenty patients with ischemic LIS. Eur Neurol. (2013) 69:207-12. doi: 10.1159/000345272

12. Ottomeyer C, Zeller J, Fesl G, Holtmannspötter M, Opherk C, Bender A, et al. Multimodal recanalization therapy in acute basilar artery occlusion. Stroke. (2012) 43:2130-5. doi: 10.1161/STROKEAHA.112. 651281

13. Schonewille WJ, Wijman CAC, Michel P, Rueckert CM, Weimar C, Mattle HP, et al. Treatment and outcomes of acute basilar artery occlusion in the Basilar Artery International Cooperation Study (BASICS): a prospective registry study. Lancet Neurol. (2009) 8:724-30. doi: 10.1016/S1474-4422(09)70173-5

14. Sairanen T, Strbian D, Soinne L, Silvennoinen H, Salonen O, Artto V, et al. Intravenous thrombolysis of basilar artery occlusion. Stroke. (2011) 42:21759. doi: 10.1161/STROKEAHA.110.605584

15. Holloway RG, Benesch CG, Burgin WS, Zentner JB. Prognosis and decision making in severe stroke. JAMA. (2005) 294:725-33. doi: 10.1001/jama.294.6.725

16. Puetz V, Sylaja PN, Coutts SB, Hill MD, Dzialowski I, Mueller P, et al. Extent of hypoattenuation on CT angiography source images predicts functional outcome in patients with basilar artery occlusion. Stroke. (2008) 39:2485-90. doi: 10.1161/STROKEAHA.107.511162

17. The TIMI Study Group. The thrombolysis in myocardial infarction (TIMI) trial. NEJM. (1985) 312:932-6. doi: 10.1056/NEJM198504043121437

18. Lindsberg PJ, Sairanen T, Nagel S, Salonen O, Silvennoinen H, Strbian D. Recanalization treatments in basilar artery occlusion-systematic analysis. Eur Stroke J. (2016) 1:41-50. doi: 10.1177/2396987316629889

19. Hacke W, Kaste M, Fieschi C, von Kummer R, Davalos A, Meier D, et al. Randomised double-blind placebo-controlled trial of thrombolytic therapy with intravenous alteplase in acute ischaemic stroke (ECASS II). Lancet. (1998) 352:1245-51. doi: 10.1016/S0140-6736(98)08020-9

20. Goyal M, Menon BK, van Zwam WH, Dippel DWJ, Mitchell PJ, Demchuk $\mathrm{AM}$, et al. Endovascular thrombectomy after large-vessel ischaemic stroke: a meta-analysis of individual patient data from five randomised trials. Lancet. (2016) 387:1723-31. doi: 10.1016/S0140-6736(16)00163-X

21. Ritvonen J, Sairanen T, Silvennoinen H, Virtanen P, Salonen O, Lindsberg PJ, et al. Outcomes and long-term mortality after basilar artery occlusiona cohort with up to 20 years' follow-up. Eur J Neurol. (2021) 8:816-22. doi: 10.1111 /ene. 14628

22. Mohr JP, Caplan LR. 26 - Vertebrobasilar disease. In: Mohr JP, Wolf PA, Grotta JC, Moskowitz MA, Mayberg MR, et al., editors. Stroke. 5th ed. Saint Louis: W.B. Saunders (2011). p. 446-84.

23. Waxman SG. The reticular formation. In: Clinical Neuroanatomy, 28e. New York, NY: McGraw-Hill Education. (2017). Available online at: https:// accessmedicine.mhmedical.com/content.aspx?bookid=1969\&sectionid= 147037558 (accessed February 7, 2021).

24. Cross III DT, Moran CJ, Akins PT, Angtuaco EE, Diringer MN. Relationship between clot location and outcome after basilar artery thrombolysis. AJNR Am J Neuroradiol. (1997) 18:1221-8.

25. Lindsberg PJ, Pekkola J, Strbian D, Sairanen T, Mattle HP, Schroth G. Time window for recanalization in basilar artery occlusion: speculative synthesis. Neurology. (2015) 85:1806. doi: 10.1212/WNL.0000000000002129

26. Alemseged F, Shah DG, Diomedi M, Sallustio F, Bivard A, Sharma $\mathrm{G}$, et al. The basilar artery on computed tomography angiography prognostic score for basilar artery occlusion. Stroke. (2017) 48:631-7. doi: 10.1161/STROKEAHA.116.015492

27. Singer OC, Berkefeld J, Nolte CH, Bohner G, Haring H-P, Trenkler $\mathrm{J}$, et al. Mechanical recanalization in basilar artery occlusion: the
ENDOSTROKE study. Ann Neurol. (2015) 77:415-24. doi: 10.1002/ana 24336

28. Jung S, Mono ML, Fischer U, Galimanis A, Findling O, de Marchis GM, et al. Three-month and long-term outcomes and their predictors in acute basilar artery occlusion treated with intra-arterial thrombolysis. Stroke. (2011) 42:1946-51. doi: 10.1161/STROKEAHA.110.606038

29. Bouslama M, Haussen DC, Aghaebrahim A, Grossberg JA, Walker G, Rangaraju S, et al. Predictors of good outcome after endovascular therapy for vertebrobasilar occlusion stroke. Stroke. (2017) 48:3252-7. doi: 10.1161/STROKEAHA.117.018270

30. Ritvonen J, Strbian D, Silvennoinen H, Virtanen P, Salonen O, Lindsberg PJ, et al. Thrombolysis and adjunct anticoagulation in patients with acute basilar artery occlusion. Eur J Neurol. (2018) 26:128-35 doi: 10.1111/ ene. 13781

31. Levy EI, Firlik AD, Wisniewski S, Rubin G, Jungreis CA, Wechsler LR, et al. Factors affecting survival rates for acute vertebrobasilar artery occlusions treated with intra-arterial thrombolytic therapy: a meta-analytical approach. Neurosurgery. (1999) 45:539-48. doi: 10.1097/00006123-19990900000025

32. Wijdicks EF, Scott JP. Outcome in patients with acute basilar artery occlusion requiring mechanical ventilation. Stroke. (1996) 27:1301-3. doi: 10.1161/01.STR.27.8.1301

33. Tsao JW, Hemphill III JC, Johnston SC, Smith WS, Bonovich DC. Initial Glasgow coma scale score predicts outcome following thrombolysis for posterior circulation stroke. JAMA Neurol. (2005) 62:1126-9. doi: 10.1001/archneur.62.7.1126

34. Schielke E, Busch MA, Hildenhagen T, Holtkamp M, Küchler I, Harms L, et al. Functional, cognitive and emotional long-term outcome of patients with ischemic stroke requiring mechanical ventilation. J Neurol. (2005) 252:64854. doi: 10.1007/s00415-005-0711-5

35. Strbian D, Sairanen T, Silvennoinen H, Salonen O, Kaste M, Lindsberg PJ. Thrombolysis of basilar artery occlusion: impact of baseline ischemia and time. Ann Neurol. (2013) 73:688-94. doi: 10.1002/ana.23904

36. Greving JP, Schonewille WJ, Wijman CAC, Michel P, Kappelle LJ, Algra A. Predicting outcome after acute basilar artery occlusion based on admission characteristics. Neurology. (2012) 78:1058. doi: 10.1212/WNL.0b013e31824e8f40

37. Woong Y, Kee KS, Wook HT, Hyun BB, Young LY, Keun KH. Predictors of good outcome after stent-retriever thrombectomy in acute basilar artery occlusion. Stroke. (2015) 46:2972-5. doi: 10.1161/STROKEAHA.115.010840

38. Strbian D, Sairanen T, Silvennoinen H, Salonen O, Lindsberg PJ. Intravenous thrombolysis of basilar artery occlusion. Stroke. (2014) 45:17338. doi: 10.1161/STROKEAHA.114.004884

39. Jadhav AP, Desai SM, Panczykowski DM, Rangaraju S, Campbell D, Ritvonen JK, et al. Predicting outcomes after acute reperfusion therapy for basilar artery occlusion. Eur J Neurol. (2020) 27:2176-84. doi: 10.1111/ene.14406

Conflict of Interest: The authors declare that the research was conducted in the absence of any commercial or financial relationships that could be construed as a potential conflict of interest.

Copyright (C) 2021 Ritvonen, Sairanen, Silvennoinen, Virtanen, Salonen, Lindsberg and Strbian. This is an open-access article distributed under the terms of the Creative Commons Attribution License (CC BY). The use, distribution or reproduction in other forums is permitted, provided the original author(s) and the copyright owner(s) are credited and that the original publication in this journal is cited, in accordance with accepted academic practice. No use, distribution or reproduction is permitted which does not comply with these terms. 